\title{
Next Generation Sequencing a Method for Identifying Genetic Mutations Associated with Spina Bifida Disorder
}

\section{Hanieh Naddaf (MSc) ${ }^{1, *}$, Arash Sattari (PhD) ${ }^{2}$, Sina Mirzaahmadi (PhD) ${ }^{3}$}

${ }^{1}$ Master of Genetic, College of Basic Science, Islamic Azad University, Zanjan Unit, Zanjan, Iran

${ }^{2}$ Post Doc of Medical Genetic, Assistant Professor, College of Basic Science, Islamic Azad University, Gorgan Unit, Gorgan, Iran

${ }^{3}$ PhD of Molecular Genetic, Assistant Professor, College of Basic Science, Islamic Azad University, Zanjan unit, Zanjan, Iran

* Corresponding Author: Hanieh Naddaf, College of Basic Science, Islamic Azad University, Zanjan Unit, Zanjan, Iran. Email: haniehnaddaf@gmail.com

\begin{tabular}{|c|c|}
\hline & Abstract \\
\hline $\begin{array}{l}\text { Received: } 16 / 10 / 2018 \\
\text { Accepted: } 15 / 12 / 2018\end{array}$ & \multirow[b]{2}{*}{$\begin{array}{l}\text { Background and Objective: Spina Bifida (SB) is a congenital } \\
\text { malformation and is a result of the failure of the closure and failure of the } \\
\text { neural tube. The causes and mechanisms of genetic involvement involved } \\
\text { in the onset of SB are still ambiguous. The present study addresses the } \\
\text { genetic variation in SB disease using Next Generation Sequencing (NGS) } \\
\text { as a powerful molecular tool for comprehensive genetic disorders studies. } \\
\text { Materials and Methods: Three complete blood samples from people with } \\
\text { spina bifida were investigated after DNA extraction using NGS-whole } \\
\text { exome sequencing (NGS-WES) method and after comparing the obtained } \\
\text { data with the control sample. The results were analyzed using Alignment } \\
\text { software (bwa), variant calling (gatk4) and Annotation (wannovar) with the } \\
\text { version of the Hg19 genome. } \\
\text { Results: Out of } 559087 \text { mutations, there are } 1205 \text { mutations of the type } \\
\text { INDELs and } 557882 \text { mutations associated with SNPs. This number of } \\
\text { mutations was compared with control samples and patients with SB. } \\
\text { Further studies ultimately identified the genes of PAX3, CUBN, MTHFR } \\
\text { and PDGFRA as more effective genes in the disease. } \\
\text { Conclusion: The NGS is a powerful method for the genetic evaluation of } \\
\text { patients with SB that can help detect genetic disorders in these patients. } \\
\text { Gene mutations found have all occurred in genes that are associated with } \\
\text { evolution in the nervous system during the fetal period. These mutations } \\
\text { should be confirmed by valid molecular methods. }\end{array}$} \\
\hline $\begin{array}{l}\text { How to Cite this Article: } \\
\text { Naddaf H, Sattari A, Mir- } \\
\text { zaahmadi S. Next Generation } \\
\text { Sequencing a Method for } \\
\text { Identifying Genetic Mutations } \\
\text { Associated with Spina Bifida } \\
\text { Disorder. Pajouhan Scientific } \\
\text { Journal. 2019; 17(2): 37-44. } \\
\text { DOI: } 10.29252 / \text { psj.17.2.37 }\end{array}$ & \\
\hline & \\
\hline
\end{tabular}




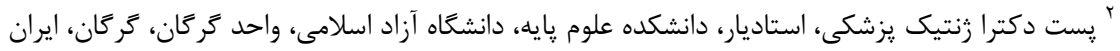

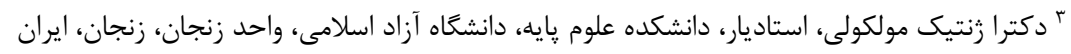
" طويسنده مسئول: هانيه نداف، دانشكده علوم پايه، دانشًاه آزاد اسلامى، واحد زنجان، زنجان، ايران. ايميل: haniehnaddaf@gmail.com

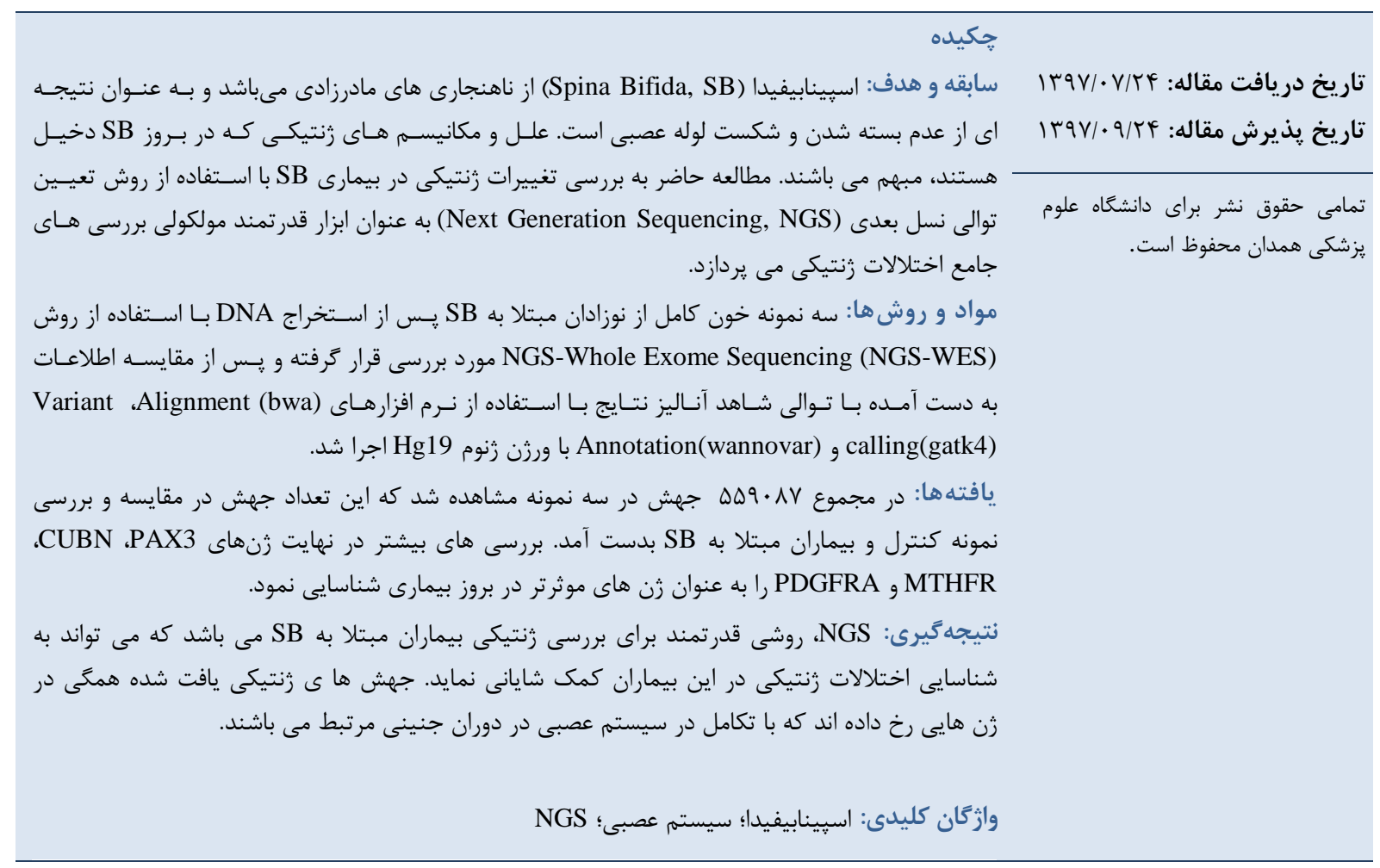

فقرات تقسيم و دو شاخه شده (Spine به معناى مهره و Bifida

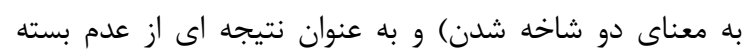

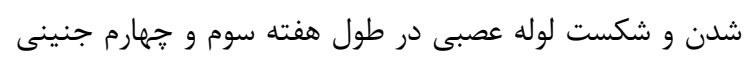

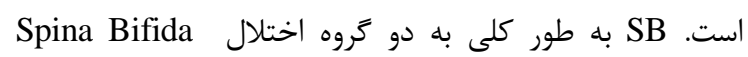
Spina Bifida Cystica (SBC) و Occulta (SBO) Myelomeningocele شامل سه كروه كه SBC Lipomeningocele geningocele (MC) ،(MMC)

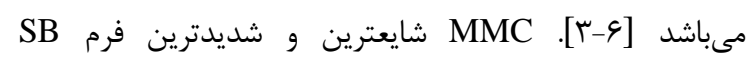

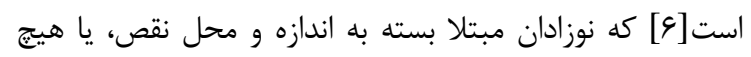

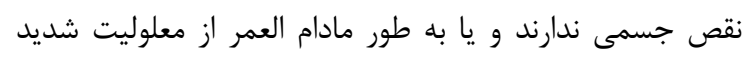

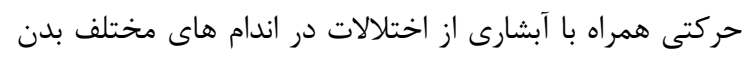

نقص لوله عصبى (Neural Tube Defect, NTDs) از شايع ترين ناهنجارى هاى مادرزادى نوزادان است كه با اختلال در عملكرد سيستم عصبى مركزى ( Central Nervous همراه مىباشد. هنگامى كة لوله عصبى در (System, CNS

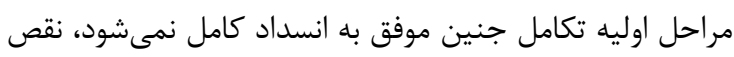

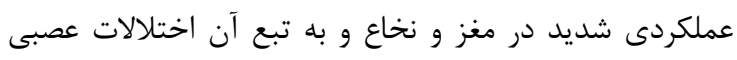

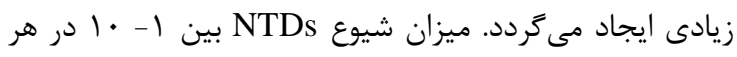

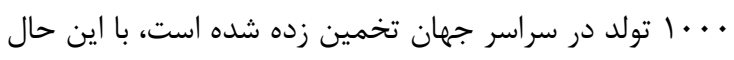

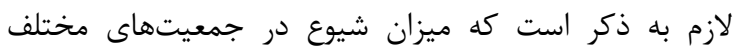

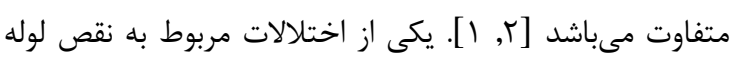

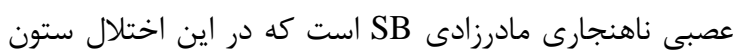


درصد از موارد با NTDs همراه است مرتبط مى باشد، اما بدون

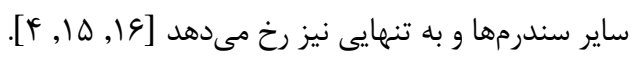

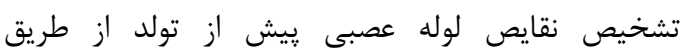

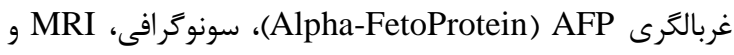

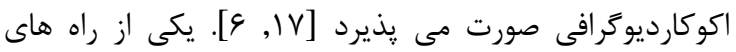

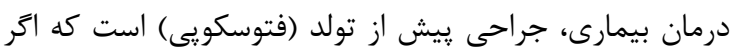

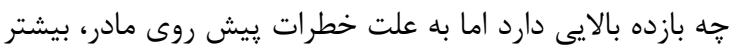

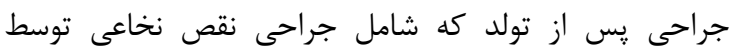
شكاف در قشر مىباشد، صورت مى يذيرد [111].

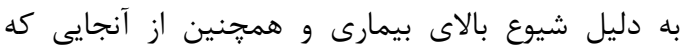

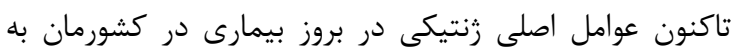

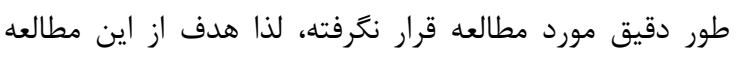

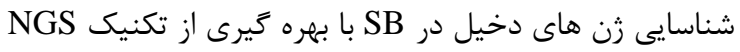

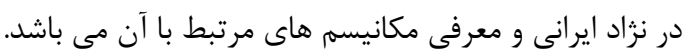

\section{مواد و روشها}

اين يزوهش توصيفى بوده و از دى ماه هوبا لغايت بهمن

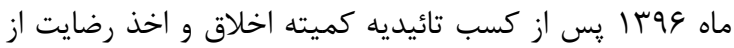
والدين نوزادان صورت يذيرفت.

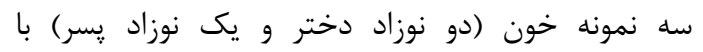

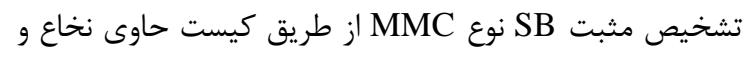

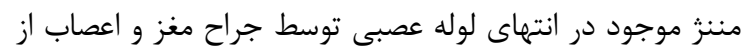
نوزادان بسترى در بخش NICU بيمارستان بعثت همدان تهيه

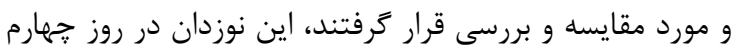
يس از تولد تحت عمل جراحى قرار كرفتند.

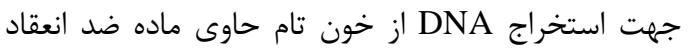
خون (EDTA)، از روش نمكىى ميلر با مقدارى دستكارى و

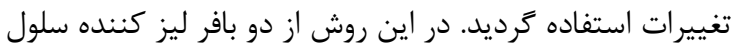

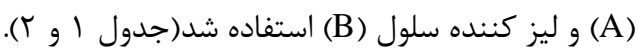

به ويزه هيدروسفالى رنج مىبرند [ه, [F].

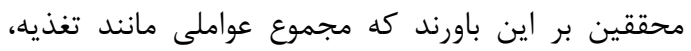

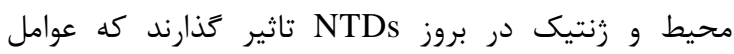

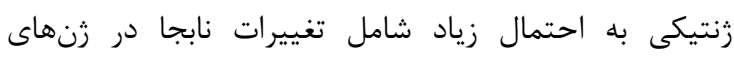

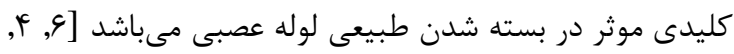

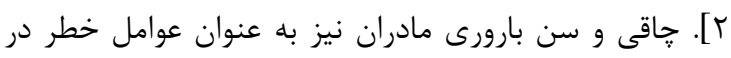

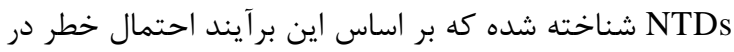

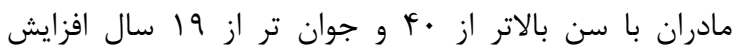

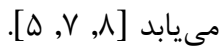
مطالعات سبب شناسى بيانگر ارتباط ميزان فولات،

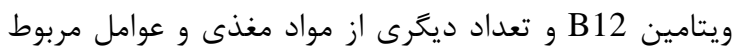

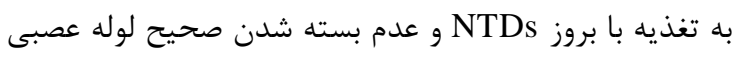

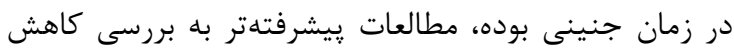

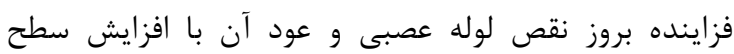

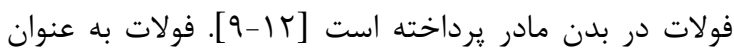

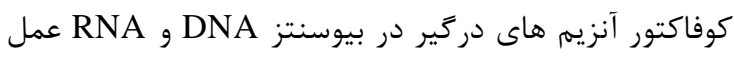

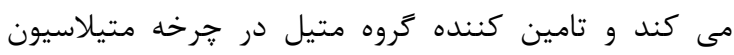

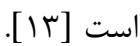
بر اساس مطالعات انجام كرفته بر روى حيوانات ثابت شده

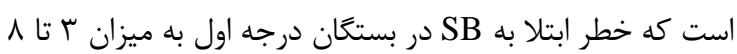

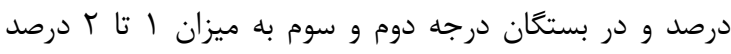

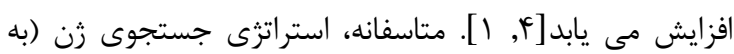

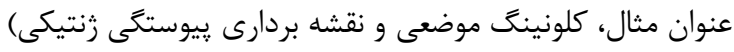

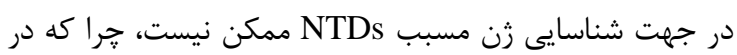

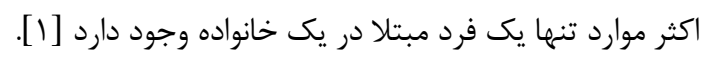
NTDs

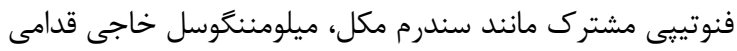

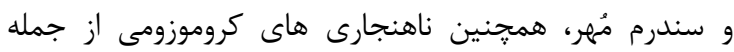

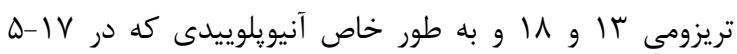

A جدول ا: تركيب بافر

\begin{tabular}{|c|c|c|c|}
\hline غلظت نهايى & ميزان مورد نياز در l+ml & غلظت & مواد \\
\hline $1 \cdot \mathrm{mM}$ & $r \mathrm{ml}$ & $\Delta / \cdot M$ & Tris- $\mathrm{HCl}(\mathrm{pH}=8)$ \\
\hline$\Delta \mathrm{mM}$ & $\mathrm{Q} / \cdot \mathrm{ml}$ & $1 \mathrm{M}$ & $\mathrm{MgCl} 2$ \\
\hline$\% 1$ & $1 \mathrm{ml}$ & - & Triton-X-100 \\
\hline$\% 11$ & $11 \mathrm{~g}$ & - & Sucrose \\
\hline- & به ميزان مورد نياز تا ml & - & آب يك بار تقطير \\
\hline
\end{tabular}

جدول r: تركيب بافر

\begin{tabular}{|c|c|c|c|}
\hline غلظت نهايى & ميزان مورد نياز در l•ml & غلظت موجود & ماده \\
\hline $1 \cdot \mathrm{mM}$ & $r \mathrm{ml}$ & $\cdot / \Delta \mathrm{M}$ & Tris- $\mathrm{HCl}(\mathrm{pH}=8)$ \\
\hline $1 \cdot \mathrm{mM}$ & $f \mathrm{ml}$ & $\cdot / r \Delta \mathrm{M}$ & EDTA \\
\hline$\% 1$ & $1 \mathrm{ml}$ & I M & سيترات سديم \\
\hline$\% 1$ & l. ml & $\%$. & SDS \\
\hline- & به ميزان مورد نياز تا ml . 1 & - & آب يك بار تقطير \\
\hline
\end{tabular}




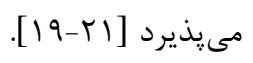

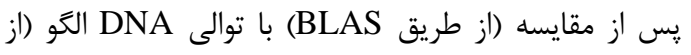

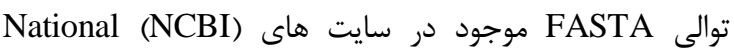
(UCSC) , Center for Biotechnology Information University of California Santa Cruz Alignment شاهد) ارزيابى بيوانفورماتيكى توسط نرم افزارهاى Annotation g (gatk4) Variant calling (bwa) با ورثن رنوم Hg19 صورت يذيرفت. (wannovar)

\section{يافته ها}

با توجه به اطلاعات بدست آمده حاصل از NGS-WES مي مئ

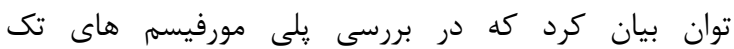
نوكلئوتيدى (Single Nucleotide Polymorphism, SNP) بران

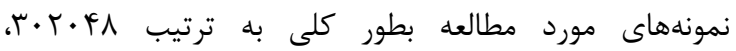

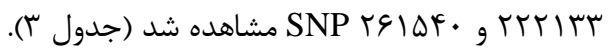

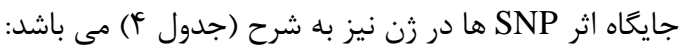
در بررسى جهش هاى Deletion and ) INDEL

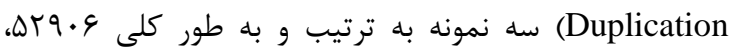

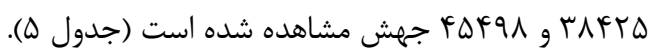

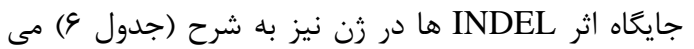

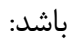

با توجه به بررسى تمامى زن هاى كزارش شده در يايكاه

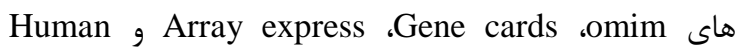
UCSC protein atlas مشترك در INDEL تعداد rال Iا زن مرتبط بان NTD

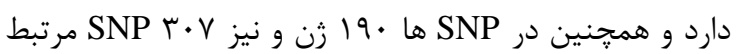

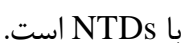

روش استخراج DNA را مىتوان به سه مرحله تقسيم

مرحله اول: تجزيه نمودن غشاى سيتويلاسمى سلولهاى

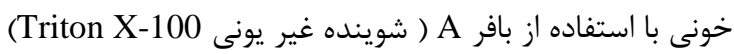

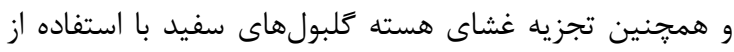

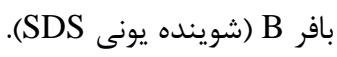

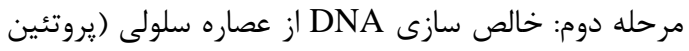
(DNA , با استفاده از نمكهاى اشباع (NaCl)

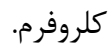
مرحله سوم: تغليظ DNA با استفاده از اتانول مطلق در درد حضور نمك و درجه برودت • آ- درجه سانتى كراد يا كمتر.

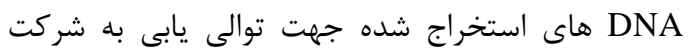

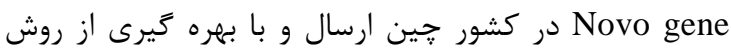

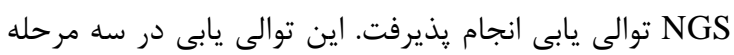

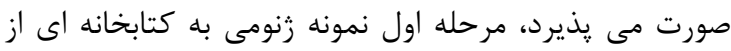

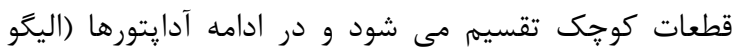

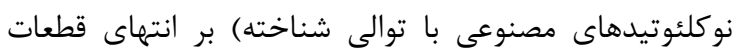

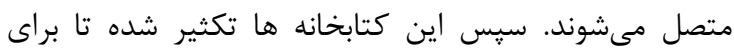

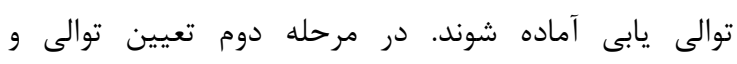

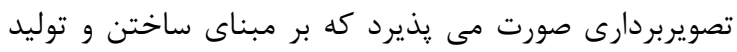

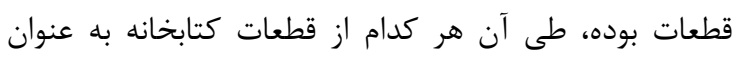

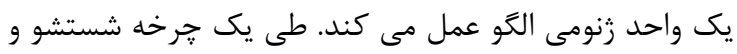

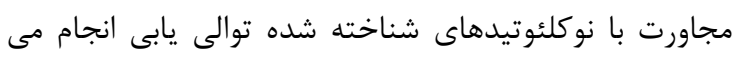

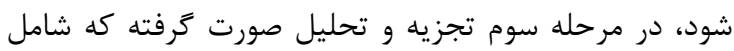

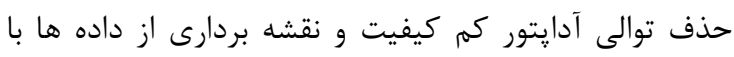

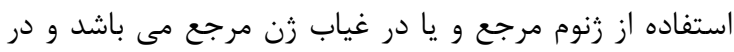

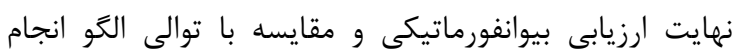

جدول بّ: داده هاى مربوط به خصوصيات SNP ها در سه نمونه

\begin{tabular}{|c|c|c|c|}
\hline شماره سه & شماره دو & شماره يك & نمونه \\
\hline TEIQT. & משITT & $r \cdot r \cdot r \wedge$ & Total SNP \\
\hline $9 \vee \cdot \wedge V$ & Q.rTr & 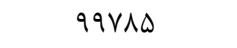 & Heterozygotes \\
\hline IEFFAT & 14191. & T. TrET & Homozygotes \\
\hline$|1| f \mid 9$ & IDFET. & $r \cdot \wedge 9 \wedge F$ & Transition \\
\hline$\Lambda \cdot|r|$ & GVDIT & $9 r \cdot q 4$ & Transvertion \\
\hline 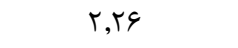 & $r, r q$ & $T, T_{\Delta}$ & ts $/ \mathrm{tv}$ \\
\hline$(/ . \vee / \Lambda ૬) r \Delta \Delta q \uparrow \wedge$ & $(/ 9 V / q F) Y / V \Delta G$. & $(/ 9 V / \wedge \Delta) r q \Delta \Delta F \Delta$ & $\mathrm{dbSNP} \%$ \\
\hline$\Delta \Delta Q T$ & FQVT & $90 \cdot r$ & Novel SNP \\
\hline THFi & TYAD & ra人d & Novel ts \\
\hline TTDI & $I \vee \wedge \Lambda$ & TDIA & Novel tv \\
\hline $1 / \Delta \varphi$ & $1 / 4 \wedge$ & $1 / \Delta \Lambda$ & Novel ts/tv \\
\hline
\end{tabular}

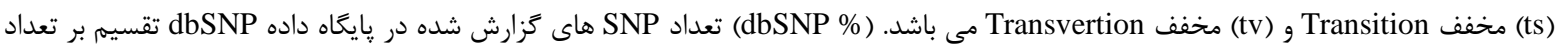


جدول fا: داده هاى مربوط به عملكرد SNP ها در سه نمونه

\begin{tabular}{|c|c|c|c|}
\hline شماره سه & شماره دو & شماره يك & 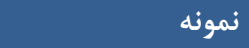 \\
\hline rTVQV & TRETI & TrIFq & CDS \\
\hline 11994 & 11099 & IIrar & synonymous_SNP \\
\hline $1 \cdot \Delta \Delta r$ & 1. FFT & $1 \cdot r \vee q$ & missense_SNP \\
\hline 19 & $\Delta F$ & vi & stopgain \\
\hline 9 & $\wedge$ & ir & stoploss \\
\hline pqr & $4 q 1$ & $\Delta \cdot r$ & unknown \\
\hline $\mid r r \Lambda \cdot r$ & 1. 9VTr & IrNAKS & intronic \\
\hline 0199 & YATA & $\Delta r q 1$ & UTR3 \\
\hline rqfi & TAIV & र१९५ & UTR5 \\
\hline$\Delta q$ & $\vee \Delta$ & 99 & Splicing \\
\hline$r i \Delta \Delta$ & rAqF & MIAD & ncRNA_exonic \\
\hline $11 V 9$. & QVTI & IT人G. & ncRNA_intronic \\
\hline 19 & $1 \cdot$ & 10 & ncRNA_splicing \\
\hline roNr & TTVG & ५१९९ & upstream \\
\hline$r \cdot \wedge \Delta$ & 1994 & Trt & downstream \\
\hline NDQYT & gFt.. & $1.944 q$ & intergenic \\
\hline TEIDF. & משוTTR & $r \cdot r \cdot r \wedge$ & Total SNP \\
\hline
\end{tabular}

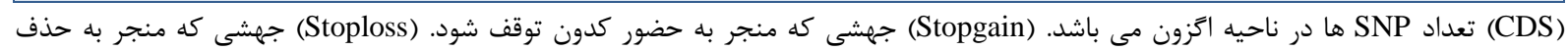

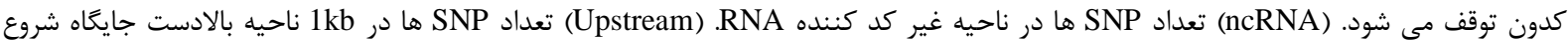

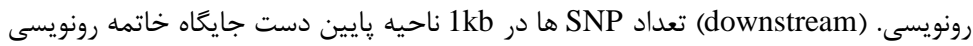

جدول ه: داده هاى مربوط به خصوصيات INDEL ها در سه نمونه

\begin{tabular}{|c|c|c|c|}
\hline شماره سه & شماره دو & شماره يك & نمونه \\
\hline$F \Delta F q \Lambda$ & TNFTD & $\Delta T Q \cdot q$ & Total INDEL \\
\hline $14 \cdot 4 \varepsilon$ & I MFTh & $|48| \mid$ & Heterozygotes \\
\hline TIFAT & rFqAV & rᄉr৭ब & Homozygotes \\
\hline$(/ . \wedge 9 / v \cdot) r \cdot \Lambda / r$ & $(/ . \wedge q / D F) M F F \cdot F$ & $(/ .9 \cdot / \cdot q)$ fVGFd & \%dbSNP \\
\hline$\forall \& \wedge \Delta$ & F.rI & DYEI & Novel INDEL \\
\hline
\end{tabular}

جدول 9: داده هاى مربوط به عملكرد INDEL ها در سه نمونه

\begin{tabular}{|c|c|c|c|}
\hline شماره سه & شماره دو & شماره يك & 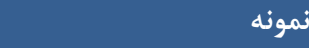 \\
\hline$v \cdot 9$ & 999 & $V \cdot V$ & CDS \\
\hline ع & 119 & $11 \cdot$ & frameshift_deletion \\
\hline 94 & 94 & $\wedge \Delta$ & frameshift_insertion \\
\hline 119 & IVA & $r \cdot V$ & nonframeshift_deletion \\
\hline 195 & 199 & 191 & nonframeshift_insertion \\
\hline f & 4 & $\wedge$ & stopgain \\
\hline • & 1 & • & stoploss \\
\hline $1 \cdot 1$ & $1 \cdot 1$ & 11. & unknown \\
\hline trtit & $r \cdot V \cdot q$ & TEFTA & Intronic \\
\hline$\wedge \wedge \Lambda$ & 948 & $1 \cdots v$ & UTR3 \\
\hline$\Delta F$. & $\Delta \cdot$ & $0 \cdot 1$ & UTR5 \\
\hline ᄉr & $\wedge 1$ & $\Delta F$ & Splicing \\
\hline
\end{tabular}




\begin{tabular}{|c|c|c|c|}
\hline & & & ادامه جدول 9. \\
\hline एव9 & reV & $\varphi .$. & ncRNA_exonic \\
\hline TrVD & INGY & ravq & ncRNA_intronic \\
\hline 1 & $\Delta$ & r & ncRNA_splicing \\
\hline$v \cdot \Delta$ & sTA & VTq & Upstream \\
\hline reV & rq1 & fis & Downstream \\
\hline IGTYA & ITMTV & 199vם & Intergenic \\
\hline rafqA & TAFTD & $0 T_{9.9}$ & Total INDEL \\
\hline
\end{tabular}

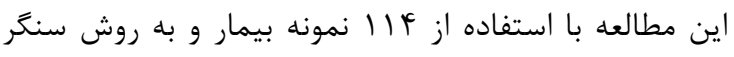

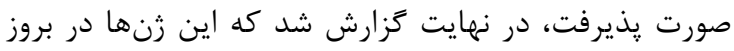

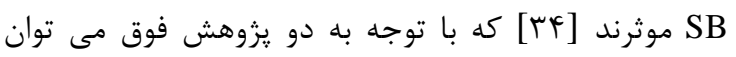

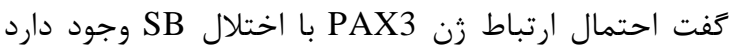
زيرا كه در مطالعه حاضر نيز اين ارتباط مشاهده شده است

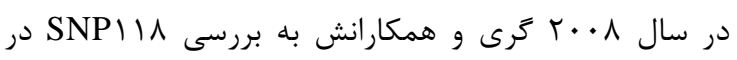

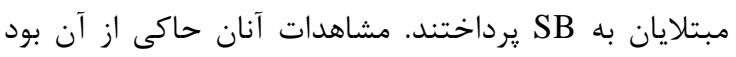

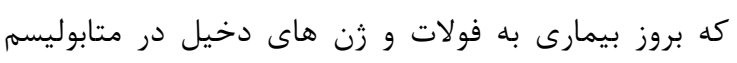

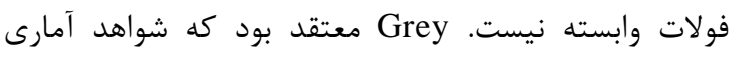

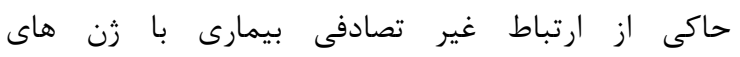

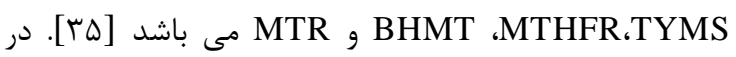

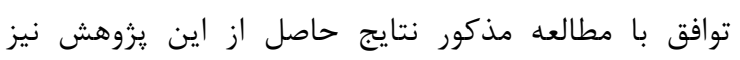
جهش در زن MTHFR مشاهده شد.

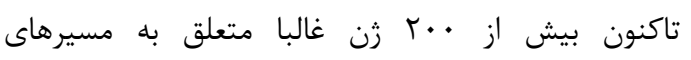

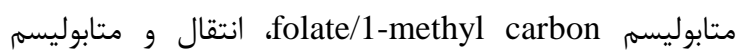

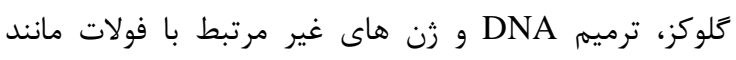

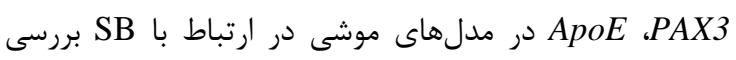

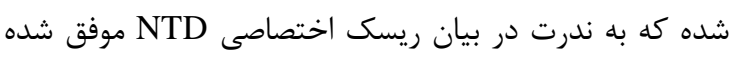

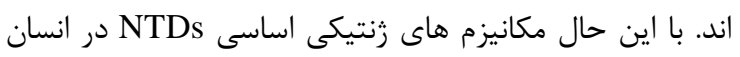

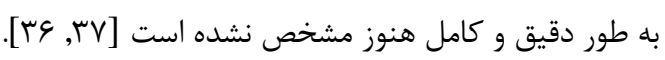

\section{نتيجه كيرى}

با توجه به اين نتايج مى توان بيان كرد كه ارتباط اين

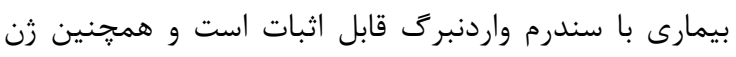

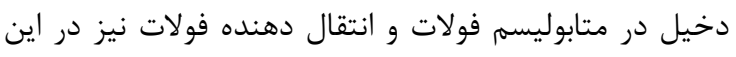

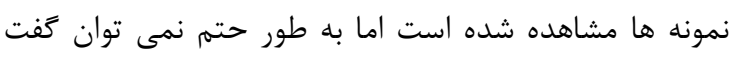

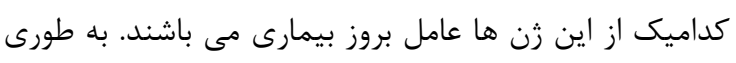

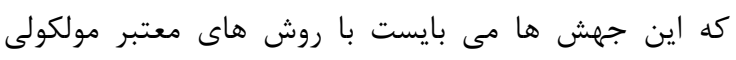

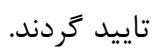

\section{تشكر و قدر دانى}

اين مقاله منتج از بايان نامه كارشناسى ارشد رشته

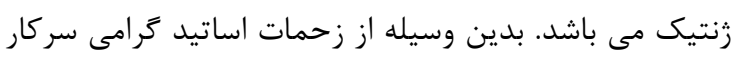

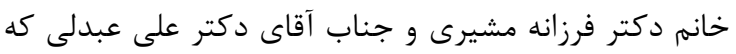

با استفاده از آناليز هاى بيوانفورماتيكى در مطالعه

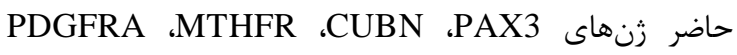
تاثيركذارتر از ساير زن هاي مان مشاهده شدند. زن (2q36.1)

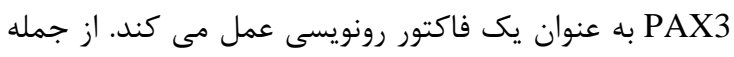

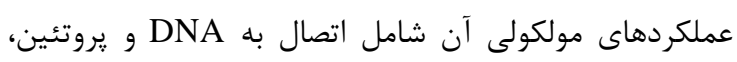

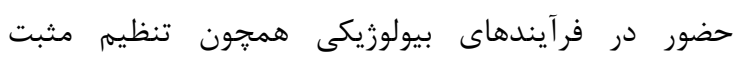

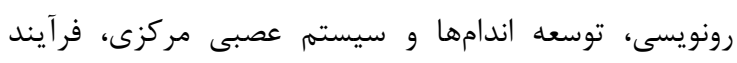

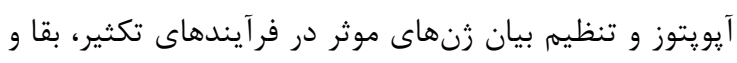

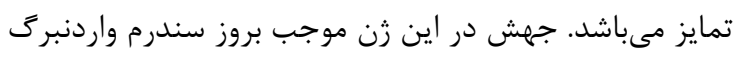

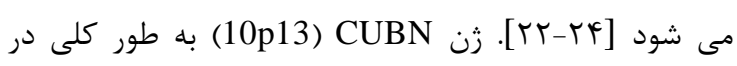

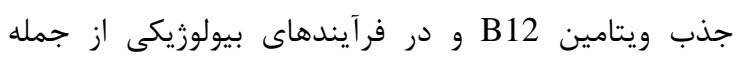

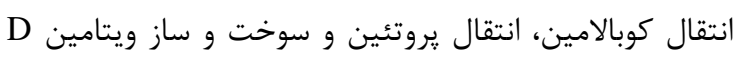

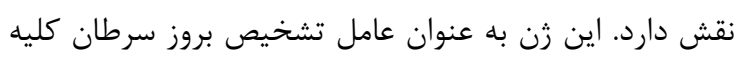

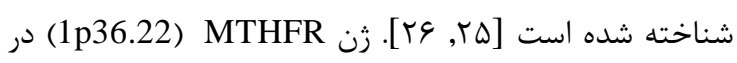

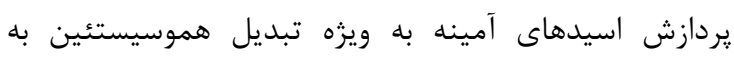

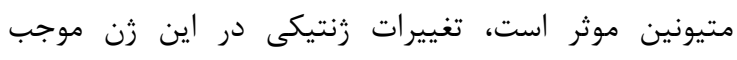

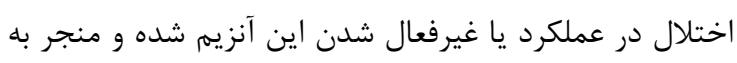

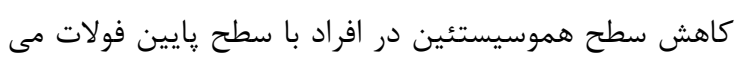

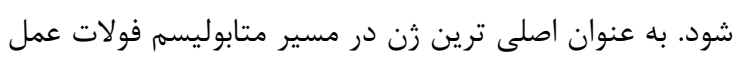

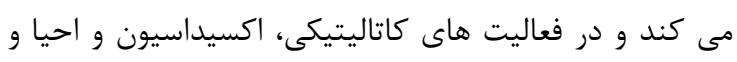

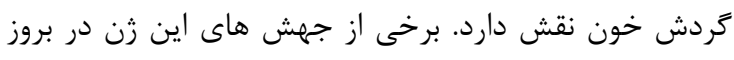

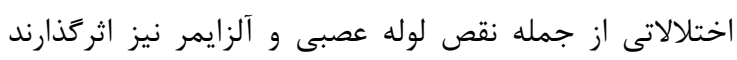

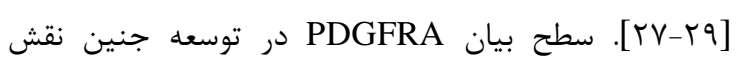

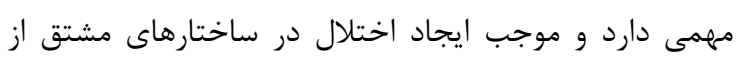

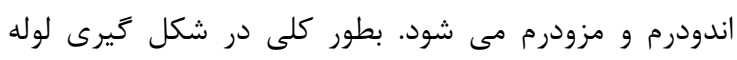

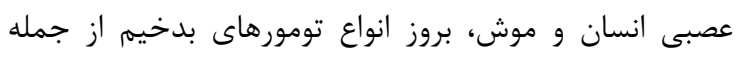

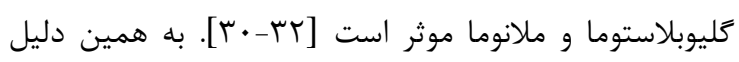

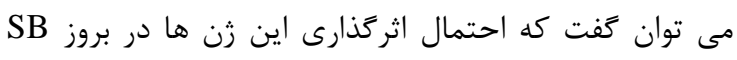

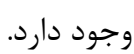
در مطالعه اى Wei Lu و همكارانش در سال و. •ب به

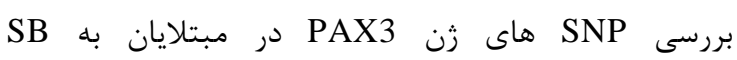

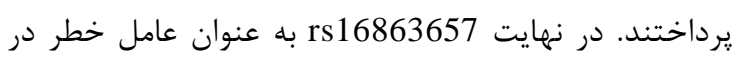

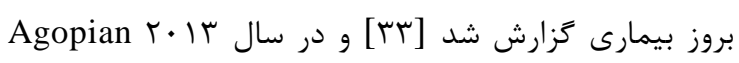

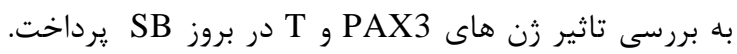




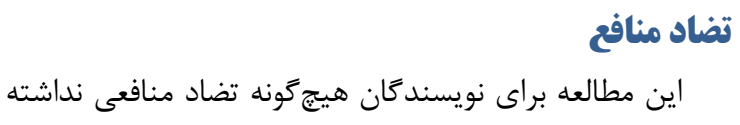

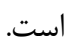

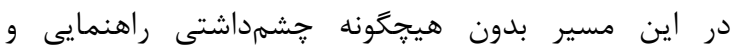

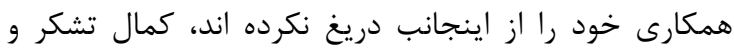

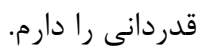

\section{REFERENCES}

1. Au KS, Ashley-Koch A, Northrup H. Epidemiologic and genetic aspects of spina bifida and other neural tube defects. Dev Disabil Res Rev. 2010; 16(1): 6-15. DOI:10.1002/ddrr.93.

2. Lei Y, Finnell RH. New Techniques for the Study of Neural Tube Defects. Adv Tech Biol Med. 2016; 4(1).

3. DeJong PM, Adams NS, Mann RJ, Polley JW, Girotto JA. Management of Lumbosacral Myelomeningocele. Eplasty. 2016; 16: ic51.

4. Detrait ER, George TM, Etchevers HC, Gilbert JR, Vekemans M, Speer MC. Human neural tube defects: developmental biology, epidemiology, and genetics. Neurotoxicol Teratol. 2005; 27(3): 515-524. DOI:10.1016/ j.ntt.2004.12.007.

5. Copp AJ, Adzick NS, Chitty LS, Fletcher JM, Holmbeck GN, Shaw GM. Spina bifida. Nat Rev Dis Primers. 2015;1: 15007.

6. Fletcher JM, Brei TJ. Introduction: Spina bifida--a multidisciplinary perspective. Dev Disabil Res Rev. 2010; 16(1):1-5. DOI: $10.1002 / d d r r .101$.

7. Waller DK, Shaw GM, Rasmussen SA, Hobbs CA, Canfield MA, Siega-Riz AM, Gallaway MS, Correa A. Prepregnancy obesity as a risk factor for structural birth defects. Arch Pediatr Adolesc Med. 2007; 161(8): 745-750.

8. Vieira AR, Castillo Taucher S. Maternal age and neural tube defects: evidence for a greater effect in spina bifida than in anencephaly. Rev Med Chil. 2005; 133(1): 62-70.

9. Kirke PN, Molloy AM, Daly LE, Burke H, Weir DG, Scott JM. Maternal plasma folate and vitamin B12 are independent risk factors for neural tube defects. Q J Med. 1993; 86(11): 703-708.

10. Molloy AM, Kirke PN, Troendle JF, Burke H, Sutton M, Brody LC, Scott JM, Mills JL. Maternal vitamin B12 status and risk of neural tube defects in a population with high neural tube defect prevalence and no folic Acid fortification. Pediatrics. 2009; 123(3):917-923. DOI:10.1542/peds.20081173.

11. Honein MA, Paulozzi LJ, Mathews TJ, Erickson JD, Wong LY. Impact of folic acid fortification of the US food supply on the occurrence of neural tube defects. JAMA. 2001; 285(23):2981-2986

12. Lumley J, Watson L, Watson M, Bower C. Withdrawn: Periconceptional supplementation with folate and/or multivitamins for preventing neural tube defects. Cochrane Database Syst Rev. 2011(4): CD001056.

13. Scott JM, Weir DG, Molloy A, McPartlin J, Daly L, Kirke P. Folic acid metabolism and mechanisms of neural tube defects. Ciba Found Symp. 1994; 181: 180-187; discussion 187-191.

14. Harris MJ, Juriloff DM. An update to the list of mouse mutants with neural tube closure defects and advances toward a complete genetic perspective of neural tube closure. Birth Defects Res A Clin Mol Teratol. 2010; 88(8):653-669. DOI:10.1002/bdra.20676.

15. Kennedy D, Chitayat D, Winsor EJ, Silver M, Toi A. Prenatally diagnosed neural tube defects: ultrasound, chromosome, and autopsy or postnatal findings in 212 cases. Am J Med Genet. 1998; 77(4): 317-321.

16. Goetzinger KR, Stamilio DM, Dicke JM, Macones GA, Odibo AO. Evaluating the incidence and likelihood ratios for chromosomal abnormalities in fetuses with common central nervous system malformations. Am J Obstet Gynecol. 2008; 199(3):285 e1-6. DOI:10.1016/j.ajog. 2008.06.100.

17. Adzick NS. Prenatal diagnosis and treatment of spina bifida. Preface. Fetal Diagn Ther. 2015; 37(3):165. DOI: org/10.1159/000375329.

18. Bevilacqua NS, Pedreira DA. Fetoscopy for meningomyelocele repair: past, present and future. Einstein (Sao

Paulo). 2015; 13(2):283-9. DOI: 10.1590/s1679-45082015 RW3032.

19. Ng SB, Buckingham KJ, Lee C, Bigham AW, Tabor HK, Dent KM, Huff CD, Shannon PT, Jabs EW, Nickerson DA, Shendure J, Bamshad MJ. Exome sequencing identifies the cause of a mendelian disorder. Nat Genet. 2010; 42(1):3035. DOI: 10.1038/ng.499.

20. Warr A, Robert C, Hume D, Archibald A, Deeb N, Watson M. Exome Sequencing: Current and Future Perspectives. G3 (Bethesda). 2015; 5(8):1543-1550. DOI: 10.1534/g3. 115.018564 .

21. Lohmann K, Klein C. Next generation sequencing and the future of genetic diagnosis. Neurotherapeutics. 2014; 11(4):699-707. DOI: 10.1007/s13311-014-0288-8.

22. Boudjadi S, Chatterjee B, Sun W, Vemu P, Barr FG. The expression and function of PAX3 in development and disease. Gene. 2018; 666:145-157. DOI: 10.1016/j. gene.2018.04.087.

23. Stuart ET, Kioussi C, Gruss P. Mammalian Pax genes. Annu Rev Genet. 1994; 28:219-236.

24. Scholl FA, Kamarashev J, Murmann OV, Geertsen R, Dummer R, Schafer BW.PAX3 is expressed in human melanomas and contributes to tumor cell survival. Cancer Res. 2001; 61(3):823-6.

25. Christensen EI, Nielsen R, Birn H. From bowel to kidneys: the role of cubilin in physiology and disease. Nephrol Dial Transplant. 2013; 28(2):274-281. DOI: 10.1093/ndt/gfs565.

26. Nykjaer A, Fyfe JC, Kozyraki R, Leheste JR, Jacobsen C, Nielsen MS, Verroust PJ, Aminoff M, de la Chapelle A, Moestrup SK, Ray R, Gliemann J, Willnow TE, Christensen EI. Cubilin dysfunction causes abnormal metabolism of the steroid hormone 25(OH) vitamin D(3). Proc Natl Acad Sci U S A. 200;. 98(24):13895-13900.

27. Fodinger M, Horl WH, Sunder-Plassmann G. Molecular biology of 5,10-methylenetetrahydrofolate reductase. J Nephrol. 2000; 13(1):20-33.

28. Trimmer EE. Methylenetetrahydrofolate reductase: biochemical characterization and medical significance. Curr Pharm Des. 2013; 19(14):2574-2593.

29. Dean L. Methylenetetrahydrofolate Reductase Deficiency, in Medical Genetics Summarie. Pratt V, McLeod H, Rubinstein W, Dean L, Kattman B, Malheiro A ,Editors. 2012; Bethesda (MD).

30. Toepoel M, Steegers-Theunissen RP, Ouborg NJ, Franke B, Gonzalez-Zuloeta Ladd AM, Joosten PH, van Zoelen EJ. Interaction of PDGFRA promoter haplotypes and maternal environmental exposures in the risk of spina bifida. Birth Defects Res A Clin Mol Teratol. 2009; 85(7):629-236.

31. Qian C, Wong CWY, Wu Z, He Q, Xia H, Tam PKH, Wong KKY, Lui VCH. Stage specific requirement of plateletderived growth factor receptor-alpha in embryonic development. PLoS One. 2017; 12(9):e0184473. DOI: 10.1371/journal.pone. 0184473 .

32. Heinrich MC, Corless CL, Duensing A, McGreevey L, Chen CJ, Joseph N, Singer S, Griffith DJ, Haley A, Town A, Demetri GD, Fletcher CD, Fletcher JA. PDGFRA activating mutations in gastrointestinal stromal tumors. Science. 2003; 299(5607):708-710

33. Lu W, Zhu H, Wen S, Laurent C, Shaw GM, Lammer EJ, Finnell RH. Screening for novel PAX3 polymorphisms and risks of spina bifida. Birth Defects Res A Clin Mol Teratol. 2007; 79(1):45-49.

34. Agopian AJ, Bhalla AD, Boerwinkle E, Finnell RH, Grove ML, Hixson JE, Shimmin LC, Sewda A, Stuart C, Zhong Y, Zhu $\mathrm{H}$,Mitchell LE. Exon sequencing of PAX3 and T (brachyury) in cases with spina bifida. Birth Defects Res A Clin Mol Teratol. 2013; 97(9):597-601. DOI: 10.1002/bdra.23163.

35. Shaw GM, Lu W, Zhu H, Yang W, Briggs FB, Carmichael 
SL, Barcellos LF, Lammer EJ, Finnell RH. 118 SNPs of folate-related genes and risks of spina bifida and conotruncal heart defects. BMC Med Genet. 2009; 10: 49. DOI:10.1186/1471-2350-10-49.

36. Greene ND, Stanier P, Copp AJ. Genetics of human neural tube defects. Hum Mol Genet. 2009; 18(R2):R113-29. DOI:10.1093/hmg/ddp347.
37. Bassuk AG, Muthuswamy LB, Boland R, Smith TL, Hulstrand AM, Northrup H, Hakeman M, Dierdorff JM, Yung CK, Long A, Brouillette RB, Au KS, Gurnett C, Houston DW, Cornell RA, Manak JR. Copy number variation analysis implicates the cell polarity gene glypican 5 as a human spina bifida candidate gene. Hum Mol Genet. 2013; 22(6):1097-1111. DOI:10.1093/hmg/dds515. 